INTERNATIONAL DESIGN CONFERENCE - DESIGN 2018

https://doi.org/10.21278/idc.2018.0436

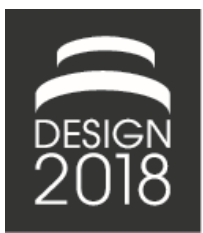

\title{
A NORMATIVE APPROACH FOR IDENTIFYING DECISION PROPAGATION PATHS IN COMPLEX SYSTEMS
}

\author{
M. Hassannezhad and P. J. Clarkson
}

\begin{abstract}
In real-world, making the right decision at the right time is very challenging. Additional complexity is related to the fact that, a change in one part may propagate across multiple subsystems and therefore, it is very difficult to predict how decisions behave. To respond this need, this paper contributes a conceptual framework to present a novel perspective on modelling decisions, so-called Decision Propagation System - DPS. It is an intelligent approach to reflect how organisational dynamics and information dependencies can affect decision propagations, thus helping make Smart decisions.
\end{abstract}

Keywords: decision making, decision automation, complexity, expert systems, dynamic decision network

\section{Introduction}

Making the right decision at the right time - in other words making a smart decision - is becoming increasingly challenging in today's environments. Advances in digitalisation along with the development of new technologies, lead to rapidly increasing amounts of information at faster speeds to be considered when making these decisions.

The trend has several consequences in business settings: first, the time from design to delivery of a product is shortening. Second, the role of the customer is becoming more important compared to the end-users and therefore their expectations (and customisations) need to be considered as early as possible during the design phase. Third, the structure of organisations (i.e., skill sets, mechanism of interactions) is changing and therefore, further considerations are required to get the right people in the right job so that they can make better decisions with respect to the status of the business.

As a result, the uncertainty of decision-making in product or service design is more significant than before and there are many upstream decisions with direct and indirect impacts on the downstream decisions. Identifying decision propagation paths in such situations would therefore be a critical issue. Even in large mature organisations, which usually have a range of pre-defined decisions at different phases, the situation does not always work as planned and many foreseen and unforeseen issues might affect the decision-making process. As a result, the composition of decisions (i.e., available decision choices) is subject to change at any time.

From this perspective, an engineering or business process can be viewed as a network of decisions whose status dynamically changes over time due to internal and external factors, such as the quantity and quality of information, or the preferences and behaviour of agents who make the decisions. As a result, as is exemplified in Figure 1, decisions in a network can be connected together in different ways, such as directly by sharing the same information, or indirectly by involving the same agent, or through their consequences. 


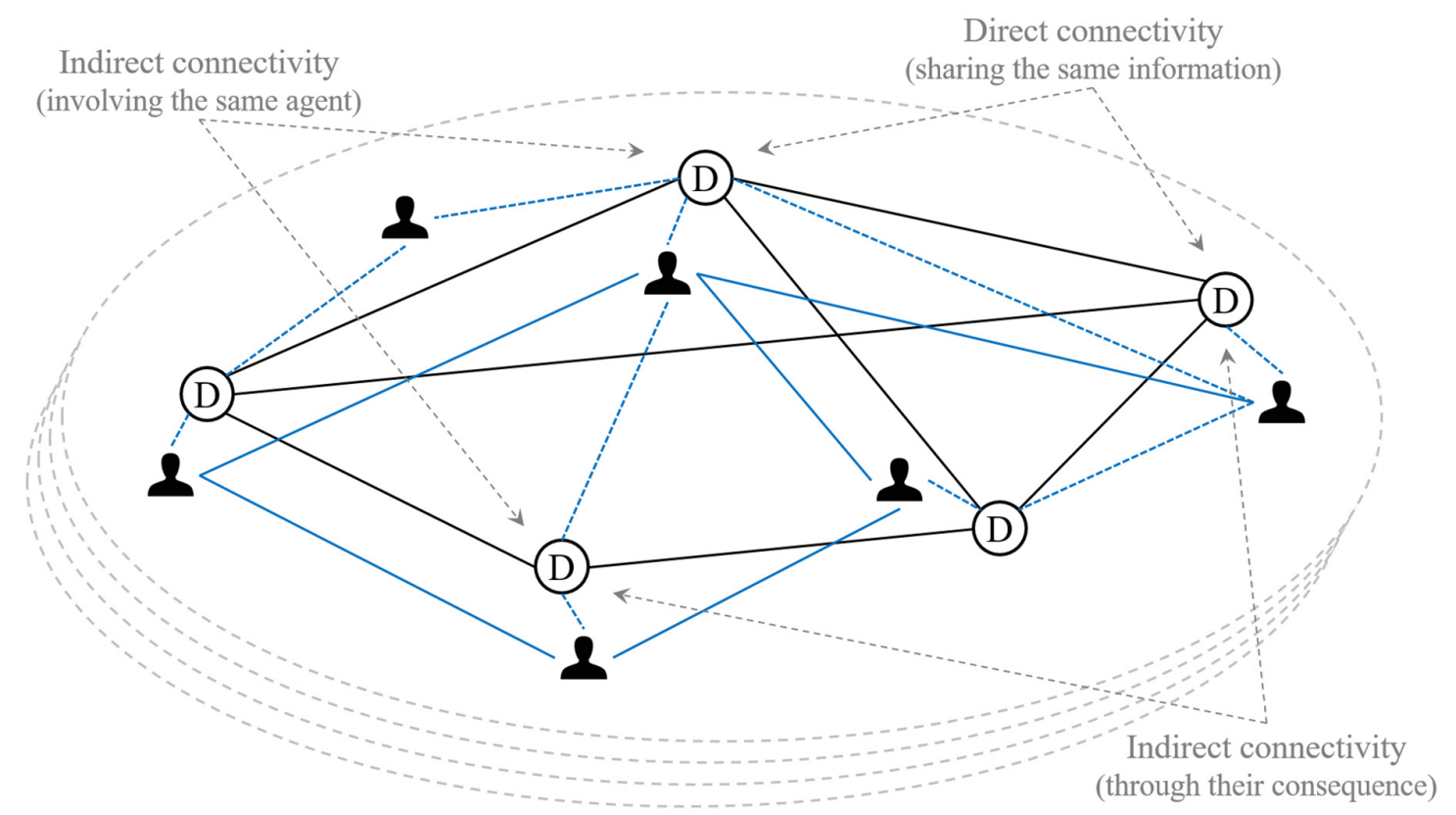

Figure 1. Decisions in a network can be connected together in different ways and with changing status over time

However, in reality of business projects, it is not simply possible to predict the behaviour of such systems (i.e., the mechanism by which decisions propagate over the time), since the consequences of a decision might go far from its local impact and globally affect other decisions - sometimes without the initial agents necessarily being aware of the implications. Therefore, it is very important for agents to get access to the right information at the right time. Additional complexity pertains to the organisational dynamics, where people with different preferences, personalities, and competencies are collaborating to make a decision.

The study of decision-making under uncertain conditions in the design field is not naive, and there have been numerous studies conducted and many models developed in the past to support decision-making of different phases of the product development processes (see Krishnan and Ulrich (2001) and Renzi et al. (2017) for overview of some of the previous studies). However, a general criticism of the majority of these models is that they have been mostly focused on expected variations (known unknowns) but unforeseen issues (unknown unknowns) have not been well investigated in the modelling.

To overcome these issues, several studies attempted to look at the term decision-making beyond the design field, by combining it with other relevant fields such as probability theory, decision theory, and game theory, for getting a better capability in representing uncertainty. Examples in the design field include the applications of Bayesian Networks (Moullec et al., 2013), Neural Networks (Ahmad et al., 2008), dynamic decision networks (Silver and De Weck, 2007), and game theory (Panchal et al., 2017). Based on previous valuable sources of knowledge, this paper contributes to building the foundations for the development of an integrated modelling framework, referred here as the Decision Propagation System - DPS. The proposed methodology considers the Design Process (DP) as a network of decisions with dependency and mutual impacts whose behaviour can be dynamically affected by the behaviour of their associated agents (e.g., designer, customer), targets (objectives), and decision consequences.

The objective is to advise managers and decision-makers on how to identify the best feasible decision of different design stages through understanding the dynamic behaviour of the decision network, i.e., the extent that early-stage decisions can affect the upcoming decisions, and which decisions (or what agents) might be most affected if something changes in the DP. In the longer term, the objective is to refine the model in a way that can train agents to adjust their behaviour according to the consequence of their decisions. 
Comparing previous dynamic decision network models in the design field, DPS aims to provide a configurable platform that is easy-to-understand while at the same time, providing rich information relating to the dynamics of decisions. This will be achieved by graphically mapping the probabilistic network of decisions, which is automatically extracted from a decision catalogue. DPS builds a holistic view of the DP and considers the social and technical aspects of the process (e.g., social- and technicalintensive consequences) in an integrated framework. Furthermore, DPS provides a simple notation to distinguish multiple types of decisions in a system, which are connected together at different levels of strength. Accordingly, the model gives information on the criticality and consistency of decisions as the outcome.

In the following of this paper, we briefly discuss aspects of decision complexity in engineering design in Section 2. Then, in Section 3, we present an overview of the literature of decision modelling in the design field and suggest that broadening the scope and looking at the other related literature is valuable. After this, modelling decision complexity in an interdisciplinary form is presented and followed by summarising the research gap in Section 4. Section 5 presents the proposed DPS framework, describing the architecture and the methodology. Finally, Section 6 discusses the application of the model in helping design problems and the outlook for further research.

\section{The complexity of decisions in engineering design}

Broadly speaking, decision-making is a cross-disciplinary term per se, and depending on the discipline, the complexity of decisions might be interpreted in different ways (Hassannezhad, 2015, p.56). In the context of engineering design, the evidence shows that the majority of the issues affecting decision complexity comes from the complexity of information (e.g., imprecision, incompleteness), organisation (e.g., subjectivity of judgement, level of competency), environment (e.g., system architecture), and the interdependencies between them. Examples of supportive references are Lewis and Mistree (1997), Marston et al. (2000), Calvano and John (2004), Aughenbaugh and Paredis (2006), Ullman (2006), Sen et al. (2010), Sen and Yang (2012), Luft et al. (2015), among many others.

Based on the review of relevant literature along with the experience of authors in discussing the issues with practitioners, a summary of factors influencing decision complexity is presented in Figure 2, and briefly described in the following. They are categorised into three groups of organisational, informational, and analytical (resulting from environmental complexity) complexity:

- Organisational complexity. Decision-making is a process during which a group of people combine their knowledge and expertise to frame a problem, evaluate the alternatives, and make a decision (Ullman, 2006). Furthermore, people show different behaviours, either individually or as a group. The difference in their behaviour can appear as a difference in their preferences and belief when making or voting a decision. In a broader setting, people learn from their prior experiences and have a tendency to copy the previous (successful) decision, regardless of its impact. This sort of differences can act as a constraint to the system preferences, especially over time, and affect expert judgements on a decision.

- Informational complexity. It is widely accepted that the decision-making process is not momentary and usually constructed through successive refinement of system's information (Hansson, 2005). From engineering design perspective, a DP is the evolution of information punctuated by decisions (Ullman, 2006) within which people collaborate and transform decisions into activities based on the available choices, belief, and systems' objectives. The context is displayed in Figure 3. Therefore, in a dynamic environment, the decisions cannot be imposed unless the reliable information is accessible to the right agents at the right time. However, the reality is often far from the ideal, and frequent changeovers (as the consequence of system dynamics) act as obstacles to this process.

- Analytical complexity. The consequence of organisational and information complexity discussed above usually appears as foreseen and unforeseen changes in the decision-making process. It can take the form of updated (new) decision choices, criteria, system goals and objectives, or a new type of connectivity between them, especially at different time slots. The influence is mutual and analytical complexity might affect the behaviour of agents who are involved in the process. Moreover, system dynamics might lead to making the conflict between 
goals and objectives (Marples, 1961), requirements (Tappeta and Renaud, 2001), or criteria (Kim and Xirouchakis, 2010).

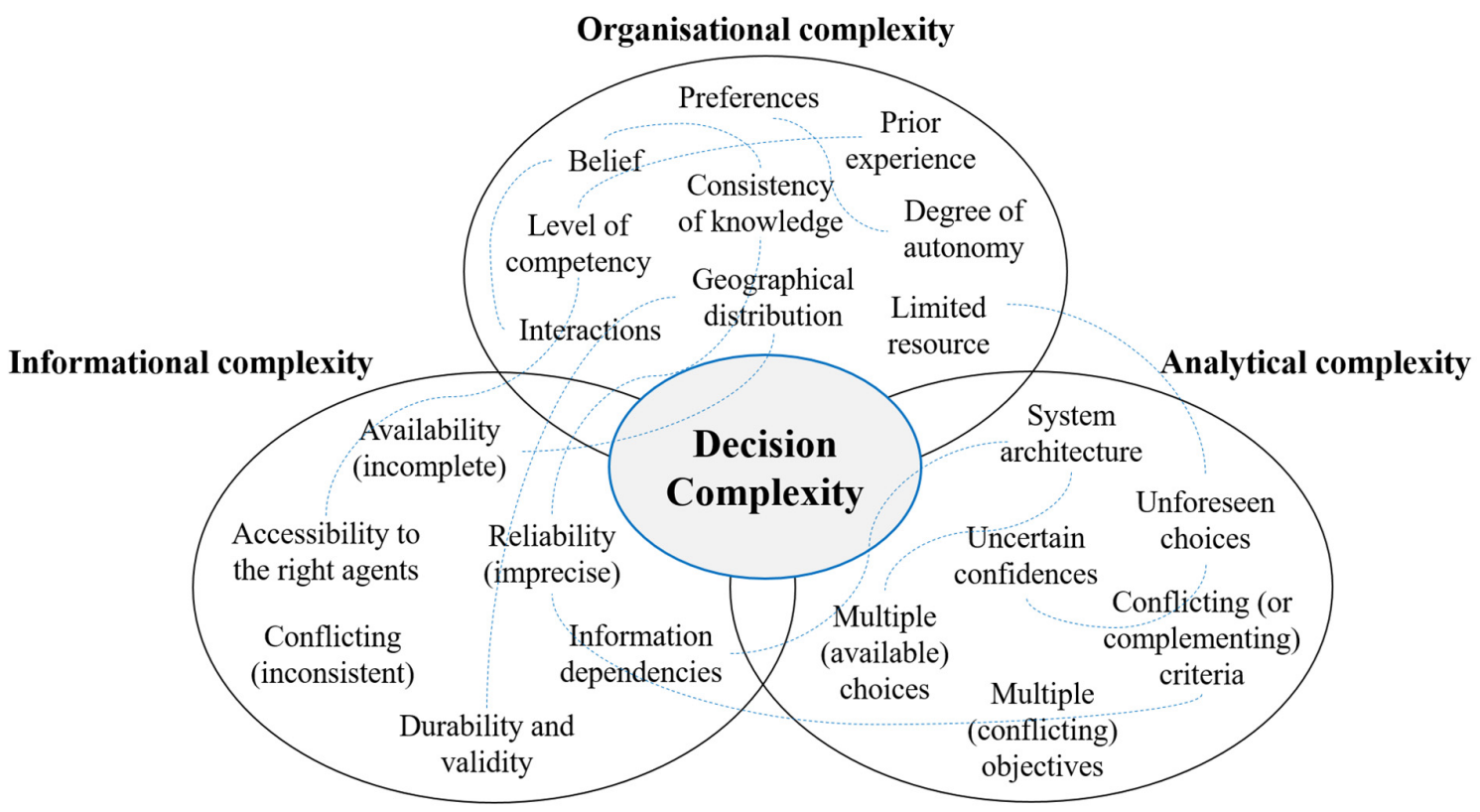

Figure 2. Decision complexity: Main aspects and the interdependencies

The next section, we present an overview of research in decision modelling in the literature of engineering design, and then expand the scope to look at the overlapping areas of engineering design with some related fields such as decision theory and probability theory.

\section{Modelling decision complexity in engineering design}

As shown in Figure 3, if we shift the focus from design activities to design decisions, by highlighting the roles of agents who make the decisions; the DP can be postulated as a series of decisions, based on the refinement of information, that assert local and global consequences on other activities. Accepting this viewpoint, decision-based modelling of DPs can be seen as an alternative to the other classes of process modelling frameworks, such as activity- and agent-based, in coping with design complexity.

Accordingly, there is a long track of research in studying decision complexity in engineering design. Marples in (1961) used two examples of plant design to study design decisions. The author then generalised the discussion and concluded that "designing is composed of a sequence of critical decisions leading from the original statement of the requirements to the specifications of the details of hardware to be manufactured" (Marples, 1961). Hespos and Strassmann (1965) proposed the Stochastic decision tree, a Monte-Carlo and GPSS simulation technique for evaluating stochastic design models based on the risk analysis of investment decisions. Other examples are Decision CPM (Crowston and Thompson, 1967), venture evaluation and review technique - VERT (Moeller and Digman, 1981), product attribute function deployment (Hoyle et al., 2006), and analytic network process (Ayag and Ozdem, 2007).

Looking at the literature for design decision modelling specifies that not all types of models are most appropriate for all design phases or for all kind of design problems (see Renzi et al. (2017) and Sen and Yang (2012) for a brief comparison). For example, as far as related to the scope of this paper, approaches such as Decision-based Design (Mistree et al., 1989), agent-based decision network (Danesh and Jin, 2001), Robust decision-making (Ullman, 2006), and collaborative DP (Fathianathan and Panchal, 2009) and (Kim and Xirouchakis, 2010) have reflected a better capability in reflecting the behaviour of agents (e.g., preference, collaboration) involved in decision-making. 


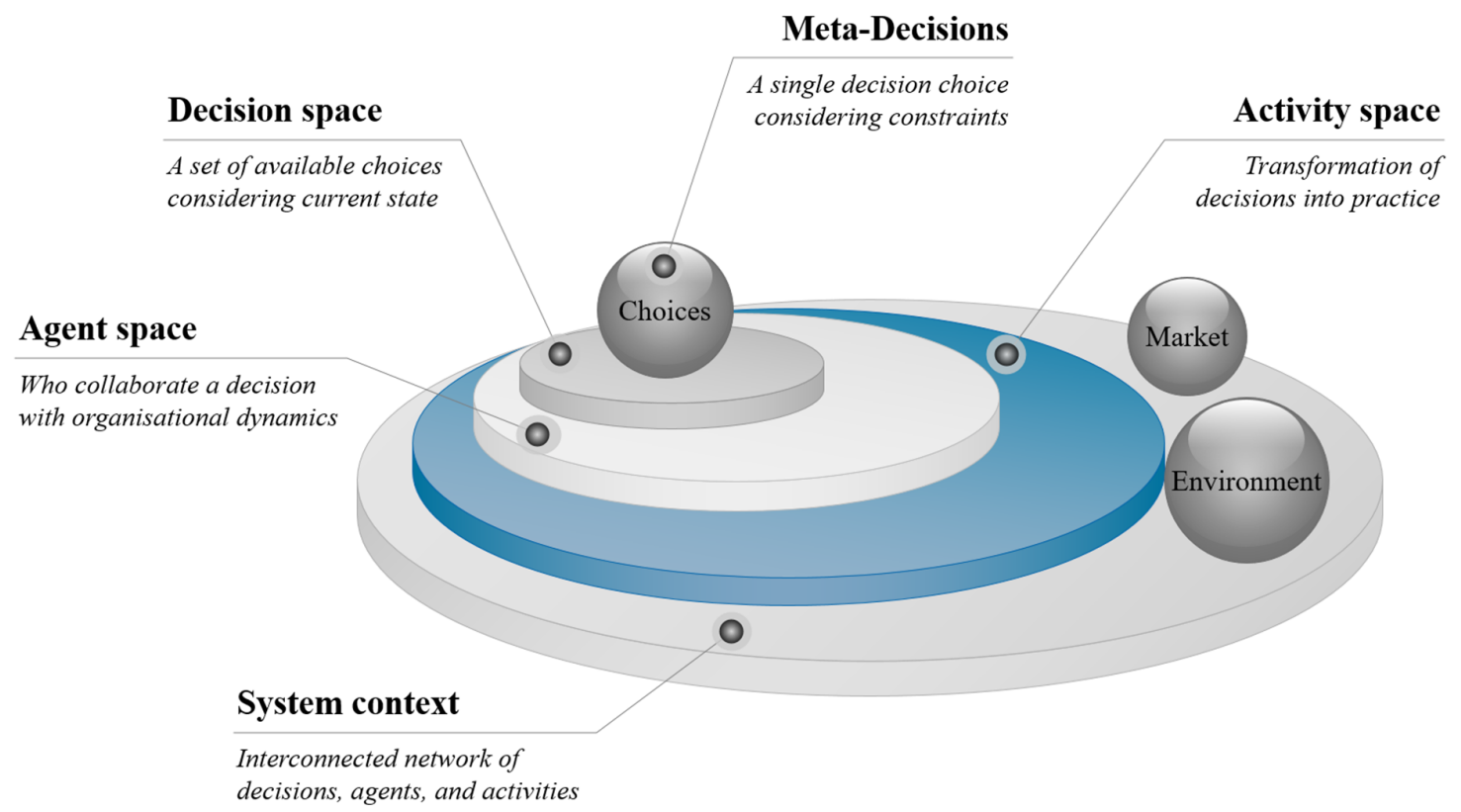

Figure 3. Modelling decision process: An approach to cope with the system complexity

Nevertheless, capturing aspects of decision complexity and the dependencies between them (Figure 2) has been a big challenge. Coping with this challenge effectively led many studies in the literature to incorporate other relevant areas of study, notably normative decision theory, and Bayesian probability theory, into the design field. An overview of research in decision modelling within the overlapping area of these fields is presented in the following.

\subsection{Modelling decision complexity in a broader context}

Broadly speaking, normative decision theory is the prescriptive area of research in decision theory, and is concerned with rationally advising on how to make the best decision through the choice of individual agents (Hansson, 2005). One of the most popular tools to support normative decision theory is decision trees, an easy-to-understand visual tool that has been supported by a number of algorithms for creating the trees in the past (Kotsiantis, 2013).

When dealing with uncertain information (i.e., incomplete, imprecise), a decision tree should be paralleled with a probability model. Hence, advancements in decision trees such as Decision Networks (DNs), also called Influence Diagrams or Bayesian decision theory, have incorporated the Bayesian probability theory into the decision theory to represent the decision-theoretic behaviour based on at least three types of variables (nodes): decision node (representing choice of agent), chance node (representing alternatives), and a value node (representing utility).

Over the years, the research community added to the DNs the capability of modelling a broader range of uncertainties (e.g., time, interdependencies, imprecision) to characterise a decision-making system that is changing or evolving over time (Russel and Norvig, 2010). Examples of such applications include Medical decision support system (Ltifi et al., 2012), Self-adaptive DN (Bencomo and Belaggoun, 2013), Partially Observable Markov Decision Processes (POMDP) (Faddoul et al., 2014), Deep Neural DN (Murthy et al., 2016), dynamic influence diagram (Shachter, 2016), as well as many applications in the area of machine learning (Ghahramani, 2015).

The design community has applied such techniques in probabilistic decision theory to characterise the inherent uncertainty of engineering design decisions. Studies such as Aughenbaugh and Paredis (2006), Panchal et al. (2009), and Thompson and Paredis (2010) addressed the value of information in dealing with design decisions complexity, and deployed the probabilistic decision theory to express uncertainty in design information (i.e., imprecision, incompleteness). Silver and De Weck (2007) focused on the 
changeability of a design system over time, and developed time-expanded DN. The authors deployed a combination of decision theory and real options to address design flexibility while switching between design configurations. Sen et al. (2010) presented a probabilistic Entropic method for uncertainty reduction through decoupling discrete design decisions.

The additional complexity of decisions is pertaining to the undeniable role of organisational dynamics in decision-making. Within a design system, designers usually negotiate a design decision, either in a centralised or decentralised structure. Uncertain information in such situations can result in uncertain decision outcomes, in which designers' confidence might vary according to the consequences (Ullman et al., 1997). This will consequently affect the information availability of downstream decisions.

Addressing these issues (i.e., the mutual impact of designer's belief and imprecise information) has been the focus of many studies in design research. For example, Moullec et al. (2013) used the Bayesian network and developed a system architecture DN, with the aim of modelling designers' knowledge (i.e., expert estimations) on the system components. Pandey and Mourelatos (2015) combined the concepts of normative decision theory and reliability block diagrams and presented the Decision Topology, as an alternative visual decision analysis tool to address traceability in assessing utility functions. Sha and Panchal (2016) presented a so-called Degree-based Decision-centric Network model, relying on the probabilistic decision theory, to investigate the impact of local interactions and preferences between individuals on the structure of a complex design system.

Most recently, Panchal et al. (2017) applied the concept of game theory in engineering design and proposed an analytical approach to support design decision-making under competition (specifically, crowdsourcing). The objective was to understand how individuals actually make decisions of acquiring information and refining the design in a competitive environment. Ming et al. (2017) refined the previous studies of Decision Support Problem (DSP) Technique (Bras and Mistree, 1991), and presented a knowledge-based platform called PDSIDES to facilitate execution and reuse of knowledge acquired by different users, namely Creator, Editor, and Implementer, in the model.

Nevertheless, research in design decision-making requires continuous effort, and in spite of substantial research in the field, several aspects of decision complexity still remain challenging for further research. Perhaps, a more versatile model might help to understand how decisions behaviour in different contexts and how multiple aspects of decision complexity affect each other.

\section{Why need a new approach?}

The primary motivation for the development of a decision-based modelling framework in this paper is to respond industry needs. As part of a collaborative project with a mature Telecoms company, the authors were asked to provide a better understanding on how decisions are made in the light of the availability of information and the pervading individuals' role and belief. For instance, one of the challenges was how we might construct feedback to the different roles considering their local consequence (job success) as opposed to the global consequence on the system behaviour.

To address these challenges facing the industry, and by reviewing the current body of literature as discussed in the previous section, the authors found some gaps as the motivation for developing a new decision-modelling framework. They are presented in the following:

- Configuration and interface. Majority of the models in the literature are based on the mathematical formulation - sometimes very complicated - which makes it difficult for nonexperts to understand and use the models in practice. A simple yet rich mapping of decision dynamics that are applicable to different problem settings is an important need. Visual representation of DNs supported by underpinning mathematical engine can be an approach to address this;

- Multiple classes of decisions. A few models distinguished different types of decisions in a design system, such as Gu et al. (2002) combining engineering and business decisions, or Lewis and Mistree (1997) distinguishing isolated, sequential, and collaborative decisions, or like Hassannezhad et al. (2017) differentiating strategic, tactical, and operational decisions. In reality, decisions are different in their nature; therefore, modelling decision ontologies can provide a more realistic view of the decision networks, while helping better understand the consistency between multiple decisions types; 
- Agents' behaviour. Research in agent-based decision-making is not naïve, but in the context of engineering design decisions, mapping individuals' belief and preferences are mostly confined to the design teams and the role of external stakeholders (such as customer) which is not appropriately defined. Furthermore, routed in the normative decision theory, each decision in the models is associated with a single individual's choice and so, the role of communication and influences on decision outcomes (decision behaviour) requires further attention;

- Quantifying consequences. Research in modelling decision consequences in engineering design is indeed poor. In addition, it has been limited to the local consequence of a decision as its direct outcome; while in reality, decisions can further, indirectly, affect other decisions in a number of ways (i.e., multiple propagation paths). Furthermore, finding the right decision choice can be rendered difficult in presence of uncertainty in the consequences of these choices;

- Information uncertainty. The review of literature in this paper showed that using the probability theory has been the dominant approach for modelling information uncertainty. However, when the probabilistic information available is vague or scarce, the elicitation of a precise probability model can be difficult, and therefore its use, questionable. Further research is nevertheless required to investigate how to elicit reliable information, in spite of some valuable studies in the design field such as Aughenbaugh and Paredis (2006) and Ahmad et al. (2008);

- Integration. An engineering design system even in its simplest form is uncertain, and the architecture of the system is subject to change at any time of the project. Central to the understanding of the dynamic behaviour of the system is to understand the mechanism of interplay between system components (e.g., decisions, choices, agents, consequences, in our case). That is only possible if we have a holistic view of the system and its components. The current body of the literature has been either focused on a particular stage of a DP (e.g., concept selection, customer requirement), or on a particular aspect of the system (e.g., information, agents, or decisions, criteria), ignoring its interaction with other aspects of the system.

Motivated by the above discussion and after reviewing the current body of the valuable literature, the next section proposes a methodology, the Decision Propagation System (DPS), as an integrated platform to model and analyse a network of decisions.

\section{The proposed Decision Propagation System (DPS)}

This section presents the proposed framework, including an overview and methodology of DPS model.

\subsection{Overview}

DPS is an integrated decision modelling and analysis platform that aims to understand the dynamics of decision architecture based on the dynamics of decision consequences, and that of individuals (agents) involved in the DP. The objective is to support managers and decision-makers with an intelligent data management system that is scalable (so that can be applied at different organisational levels), updatable (so that can react to any change in information), and easy-to-understand (so that can be used by nonexperts).

The modelling in DPS is based on graphically mapping the network of decisions that are automatically extracted from a decision catalogue. Therefore, it has two fundamental parts: Catalogue and Workplace. The DPS catalogue acts as a data management system that contains multiple classes of information relating to the decisions and their consequences, associated agents. The input of the catalogue directly comes from the users and can be modified at any time of the project (e.g., with respect to new evidence). The workplace is the place where the probabilistic network of decisions and their associated agents is drawn. This can be performed by selecting the appropriate notation from the template and then simply dragging the decisions from the catalogue and drop them in the workplace. This is shown in Figure 4. The idea behind the network-based representation of the design decisions and their consequences is to provide an intuitive approach for formulating the underlying probabilistic model, whilst also providing a formal mathematical model for analysing that representation. We use the Bayesian theory (Pearl, 1988) to build the underpinning mathematical engine, and to capture aspects of information uncertainty in the model. The idea is that, as mentioned before, decisions of a system can be connected together in three 
different ways: either directly or indirectly. These aspects of decision connectivity will be used to obtain the strength of dependencies between any two decisions, and then to help compute the Cumulative Confidence Ratio $(C C R)$ of a decision. Eventually, the information will be used for the classification of decisions (i.e., their consistency and criticality) during the simulation and analysis.

\subsection{The DPS methodology}

The outline of the DPS methodology is presented in following Figure 4. According to the figure, DPS has four main phases:

(1) Create decision catalogue. The first phase involves decomposing the system into its building blocks, which in this case are the main decision points, alternatives (associated choices), agents (including their roles and preferences), and possible consequences of decisions (targets). For example, if you are travelling between two cities, a decision point is the travelling type and the associated choices are car, coach, and train. The associated agents are the traveller, other passengers, coach driver, bus driver, and the road traffic. Possible consequences might be savings in cost, travel duration (delay), and comfortability, for instance.

In fact, in this phase, the user classifies and converts the raw data obtained from experts' opinion and documents this into the input information of the model, whose full list will appear in the catalogue interface. The catalogue acts as a database in DPS and can be refined at any time by the user(s). The information provided in this phase will later be used for graphical representation of the decision network (in phase 2), and for mathematical formulation of the underlying probabilistic model (in phase 3).

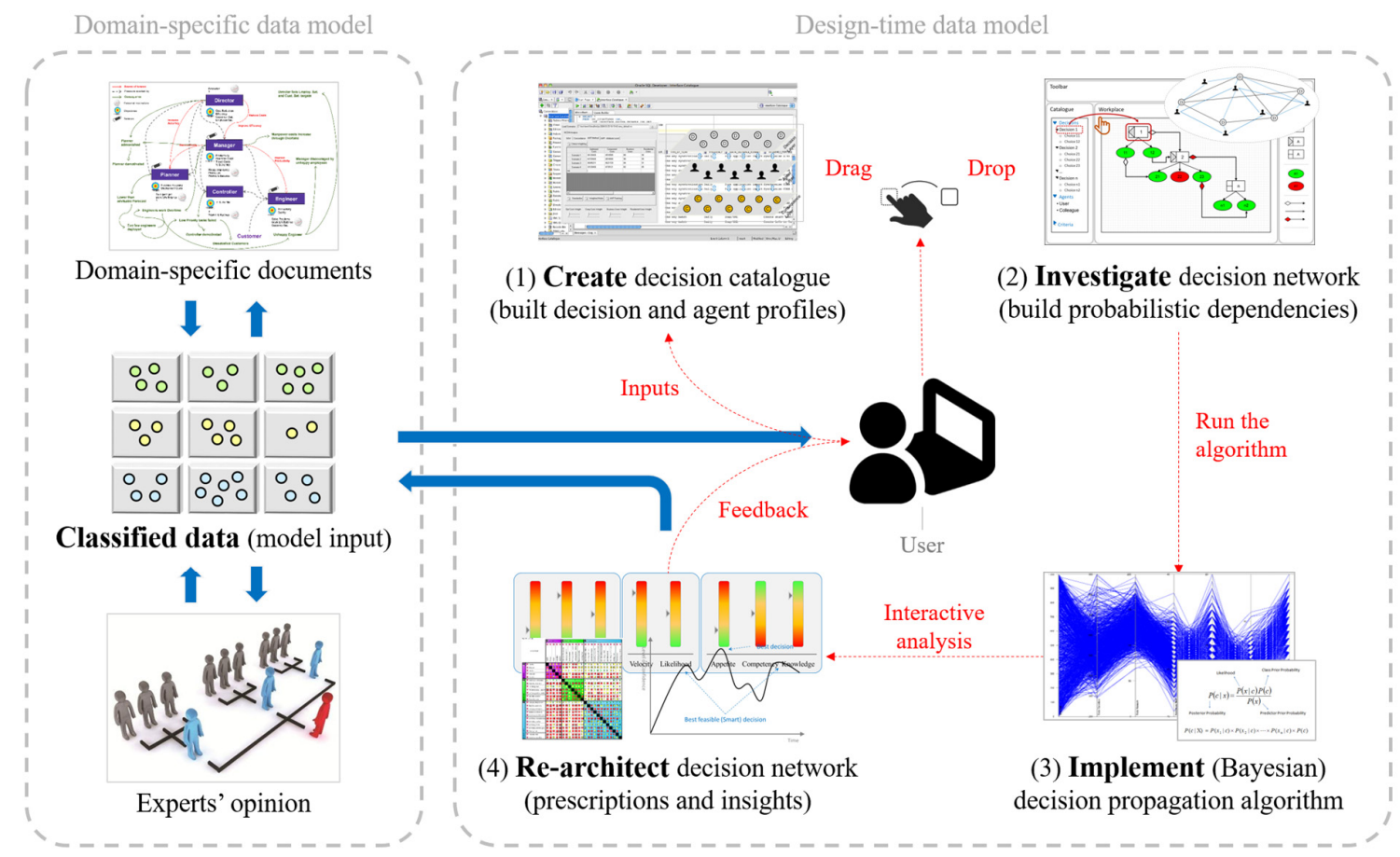

Figure 4. The proposed DPS methodology: The design model and the main phases

(2) Investigate decision network. The decision catalogue does not capture the connectivity between input information directly. In this phase, the user can use the information and make a decision network by selecting the appropriate node from the decision template and simply dragging the item from the catalogue and dropping it into the workspace. The idea is to enable the DPS engine to (intelligently) realise the information type in the catalogue and convert it into the corresponding node in the workplace. Accordingly, any change in the input information will be automatically applied to the workplace.

After that, the user can select the appropriate type of connectivity from the template to build the networks. In fact, the template provides a range of notations that enable the user to model multiple classes of decisions, agents, consequences, and multiple types of connectivity between them. An 
example of DPS notations is shown in Figure 5(a-b). The connectivity profile in the template is based on the premise that decisions can be connected together in three different aspects. These aspects are indicated in Figure 5(b).

Accordingly, DPS provides three different views of a decision process: the main view of the network of decisions, and two additional views relating the network of decisions-agents, and decisionsconsequences. It is assumed that agents are the main channel of information processing whose decision cannot only affect their corresponding activities (local consequences); it can also affect other decisions and the system behaviour (global consequence). Consequences therefore have a broad meaning in DPS and cover all issues relating to the time, cost, productivity, customer satisfaction, etc.

By building the decision network, the next step is to indicate the strength of connectivity. It is central to building the probabilistic Bayesian network later. In DPS, the strength of connectivity is a weighted vector of three aspects of decision connectivity that we discussed before, based on the dependency of information, agent, and consequence (Figure 5(b-c)).

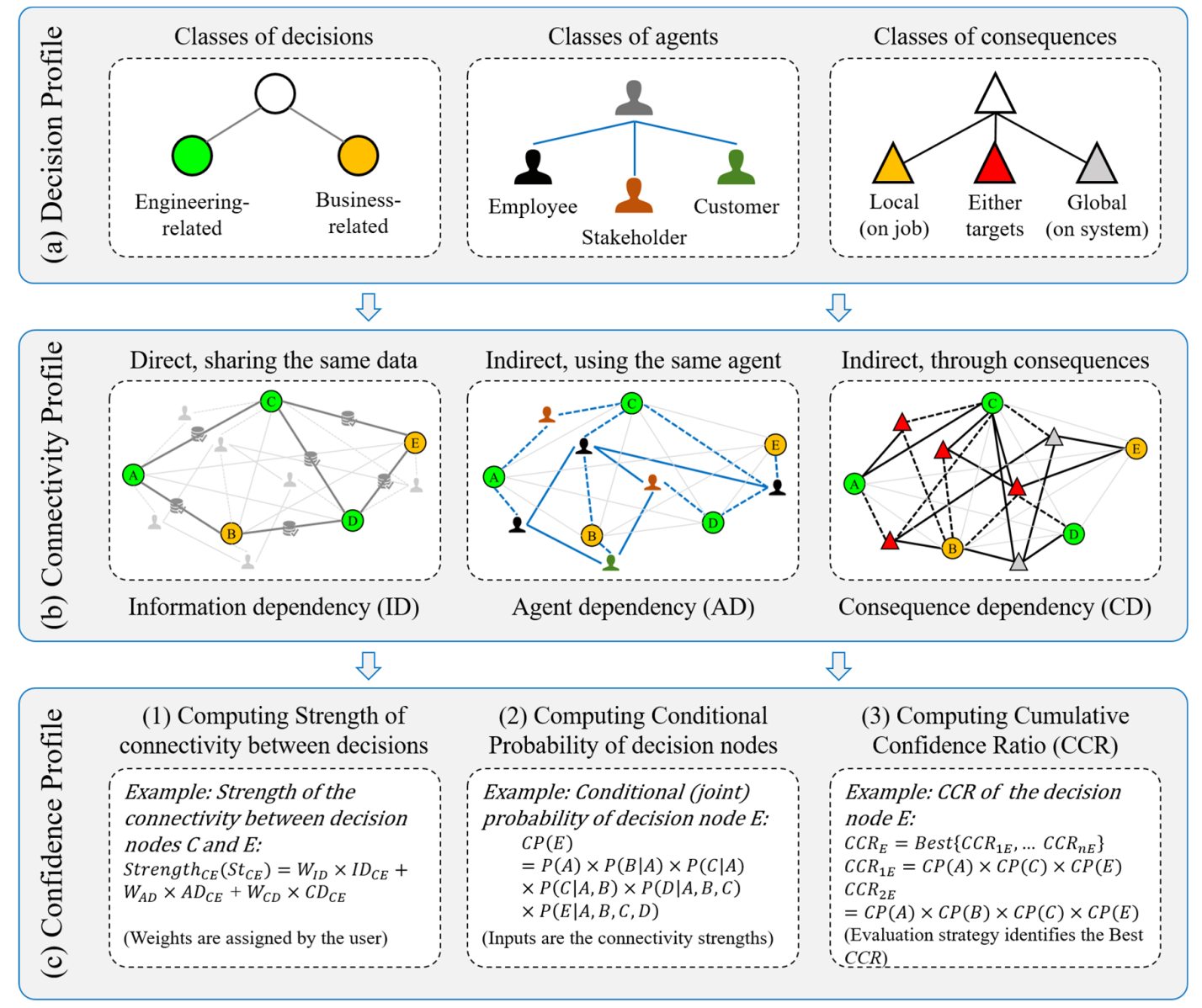

Figure 5. The logic of DPS: Indirect propagation based on direct connectivity

The objective is to reduce the impact of experts' judgement in defining the probabilities. It is particularly helpful for modelling of a highly interconnected network of decisions, or when there is not enough evidence (information) to specify the strengths (e.g., new processes). In the proposed model, the experts just need to specify the weight of elements such as agents' role, consequence types, etc.

(3) Implement decision propagation algorithm. Given the strength of connectivity, the model computes the Conditional Probability $(C P)$ for each decision node (Figure 5(c)). This will be accomplished by using the well-known Bayesian algorithm (Pearl, 1988). CP is a measure of certainty that a decision can influence other decisions in the network, and will then be used for computing the Cumulative Confidence Ratio $(C C R)$. CCR is a unique value for each decision node indicating the 
criticality of the path towards the node. Its functionality is comparable to the utility nodes in DNs, while unlike the typical DNs, there is a confidence node for each decision choice. There might be several CCRs for a decision node with different values. In fact, CCR represents the indirect impact of decision nodes based on their direct connectivity. The underlying idea is to provide a mechanism to analyse multiple paths between any two nodes, thus helping to identify the low-risk leverages. The outcome of this phase would be a range of visualisation tools to reflect the dynamics of the system.

(4) Re-architecting decision network. It is already mentioned that the DPS interface reacts to any change in the input information in the catalogue and will be updated accordingly. This unique feature can be used after implementation and can accommodate the simulation outcomes to refine (and optimise) the original model. As such, analysing the propagation paths between decisions can enable the user to understand the criticality and consistency of decisions so far. Besides, the interactivity and real-time sensitivity analysis of the simulation model enables the user to apply a broad range of scenario analysis to understand the impact of different architectures (changing connectivity), strategies (deactivating or changing consequences as the result of change in available choices), or beliefs (changing weights) and preferences that are come from the target audience of the model.

\section{Discussion and outlook: How DPS can help design?}

Quantifying the consequence of decision-making is not always straightforward. Agents involved in a process each has a specific set of targets and objectives as well as their own motivation and preferences. At the strategic level, managers are hoping to achieve a balance between costs and customer satisfaction, while at the operational level, increasing employees' productivity (through mitigating stress and demotivation) and keeping work-life balance would be a priority for decision-makers.

The local target of a role might be a global target for another role. Work-life balance is a high-priority for an operator, but a low-priority for a director. At the end of the day, customer satisfaction might be achieved (number of which make managers happy) but at the cost of unhappy operators and line managers, due to more overtime jobs and raised stress, pressure, and rework.

Decision architecture of such systems is inherently complex, and understanding the drivers behind the decisions is therefore very valuable, both for research and for practice. In light of the current body of literature reviewed in Section 3, in this paper, the authors presented the foundations of developing an integrated modelling approach to address needs of research and industry (Section 4). Rather than looking at a particular design stage, the proposed DPS model considers the DP as a whole to help to design a system architecture. Hence, the main audience of the model are medium- and high-level managers whose decisions are less sequential and propagate across organisational disciplines.

From a research perspective, DPS presents an alternative way of modelling and analysing decisions under uncertainty. The rationale behind the proposed model has the potential to address almost all of the research gaps presented in Section 4 to some extent, with more concentration on the configuration, multiple classes of decisions, and quantifying consequences. However, full consideration of the research gaps requires a fully integrated model, which is not simply possible and has been the aim of many studies over the past decades.

In terms of functionality, owing to its configurable structure, the model can deal with both expected variations and unforeseen issues (such as new evidence). This feature together with the functionality of Bayesian theory in mapping complex networks can bring scalability and updatability in a single framework. DPS distinguishes multiple classes of information (decisions, agents, and consequences) and uses this semantics and the connectivity between them to understand the criticality and consistency of decisions. In order to reduce experts' judgement (as the main source of information uncertainty in decision theory), the authors proposed a heuristic to compute decision confidence, which can determine the likelihood of (top-down and bottom-up) propagations in the network.

With regards to practitioners, the proposed model provides a more realistic view of decisions and decision-making in an organisation. Rather than proposing the optimal solution, DPS can offer the most feasible scenarios of decisions through a broad range of what-if questions. For example, how can a change in a decision affect other decisions and whom might be mostly affected. This will eventually help identify the most influential decisions, consequences, and agents of the system. 
However, the current version of DPS does not directly capture time-related uncertainty and the user should apply any change in information into the model. Dealing with imprecise information requires a more robust algorithm and can be considered as part of the future directions. The proposed model is sensitive to the knowledge of the main decision points of the system as well as existing roles and their targets. Therefore, its functionality in designing decision architecture of very new DPs might be affected. Nevertheless, the DPS prototype is under development at the time of writing of this paper and several iterations might be required to mature the original idea that presented in this paper.

\section{References}

Ahmad, A., Basir, O., Hassanein, K. and Azam, S. (2008), “An Intelligent Expert Systems’ Approach to Layout Decision Analysis and Design under Uncertainty”, In: Phillips-Wren, G., Ichalkaranje, N. and Jain, L.C. (Eds.), Intelligent Decision Making: An AI-Based Approach, Springer, Berlin, Heidelberg, pp. 321-364.

Aughenbaugh, J. and Paredis, C. (2006), "The Value of Using Imprecise Probabilities in Engineering Design", Journal of Mechanical Design, Vol. 128 No. 4, pp. 969-979. https://doi.org/10.1115/1.2204976

Ayag, Z. and Ozdem, R. (2007), "An analytic network process-based approach to concept evaluation in a new product development environment”, Journal of Engineering Design, Vol. 18 No. 3, pp. 209-226.

Bencomo, N. and Belaggoun, A. (2013), "Supporting decision-making for self-adaptive systems: From goal models to dynamic decision networks", In: Doerr, J. and Opdahl, A.L. (Eds.), Requirements Engineering: Foundation for Software Quality, Springer, Berlin, Heidelberg, pp. 221-236. https://doi.org/10.1007/978-3642-37422-7_16

Bras, B. and Mistree, F. (1991), "Designing design processes in decision-based concurrent engineering”, Journal of Materials \& Manufacturing, Vol. 100, pp. 451-458.

Calvano, C. and John, P. (2004), "Systems engineering in an age of complexity", Systems Engineering, Vol. 7 No. 1 , pp. $25-34$

Crowston, W. and Thompson, G. (1967), "Decision CPM : A Method for Simultaneous Planning, Scheduling, and Control of Projects", Management Science, Vol. 15 No. 3, pp. 407-426.

Danesh, M. and Jin, Y. (2001), "An Agent-Based Decision Network for Concurrent Engineering Design", Concurrent Engineering: Research and Applications, Vol. 9 No. 1, pp. 37-47.

Faddoul, R., Raphael, W., Soubra, A. and Chateauneuf, A. (2014), "Incorporating Bayesian Networks in Markov Decision Processes", Journal of Infrastructure Systems, Vol. 19 No. 4, pp. 415-424.

Fathianathan, M. and Panchal, J. (2009), "Incorporating design outsourcing decisions within the design of collaborative design processes", Computers in Industry, Vol. 60 No. 6, pp. 392-402.

Ghahramani, Z. (2015), "Probabilistic machine learning and artificial intelligence", Nature, Vol. 521, pp. 452459. https://doi.org/10.1038/nature14541

Gu, X., Renaud, J., Ashe, L., Batill, S., Budhiraja, A. and Krajewski, L. (2002), "Decision-Based Collaborative Optimization", Journal of Mechanical Design, Vol. 124 No. 1, pp. 1-13. https://doi.org/10.1115/1.1432991

Hansson, S.O. (2005), Decision Theory: A Brief Introduction, Royal Institute of Technology.

Hassannezhad, M. (2015), Model-Based Support for Management of Engineering Design Processes, PhD thesis, Politecnico di Torino.

Hassannezhad, M., Cassidy, S. and Clarkson, P. (2017), "Dynamic modelling of relationships in complex service design systems", ICED 17 - International Conference on Engineering Design, Vancouver, Canada, 2017.

Hespos, R. and Strassmann, P. (1965), "Stochastic Decision Trees for the Analysis of Investment Decisions", Management Science, Vol. 11 No. 10, pp. B244-B259.

Hoyle, C., Kumar, D. and Chen, W. (2006), "Product attribute function deployment (PAFD) for decision-based conceptual design”, ASME 2006 International Design Engineering Technical Conferences and Computers and Information in Engineering Conference, Volume 4a, Philadelphia, PA, USA, September 10-13, 2006.

Kim, D. and Xirouchakis, P. (2010), "CO2DE : a decision support system for collaborative design", Journal of Engineering Design, Vol. 21 No. 1, pp. 31-48.

Kotsiantis, S. (2013), “Decision trees: A recent overview”, Artificial Intelligence Review, Vol. 39 No. 4, pp. 261283.

Krishnan, V. and Ulrich, K. (2001), "Product Development Decisions : A Review of the Literature", Management Science, Vol. 47 No. 1, pp. 1-21.

Lewis, K. and Mistree, F. (1997), “Collaborative, sequential, and isolated decisions in design”, 1997 ASME Design Engineering Technical Conferences, Sacramento, California, September 14-17, 1997, pp. 1-13.

Ltifi, H., Trabelsi, G., Ben Ayed, M. and Alimi, A.M. (2012), "Dynamic Decision Support System Based on Bayesian Networks Application to fight against the Nosocomial Infections", International Journal of Advanced Research in Artificial Intelligence, Vol. 1 No. 1, pp. 22-29. 
Luft, T., Schneider, S. and Wartzack, S. (2015), “A Methodical Approach To Model and Map Interconnected Decision Making Situations and Their Consequences", ICED 15 - International Conference on Engineering Design, Milan, Italy, 2015, pp. 1-12.

Marples, D. (1961), “The Decisions of Engineering Design”, IRE Transactions on Engineering Management, Vol. EM-8 No. 2, pp. 55-71.

Marston, M., Allen, J. and Mistree, F. (2000), “The decision support problem technique: Integrating descriptive and normative approaches in decision based design", Engineering Valuation and Cost Analysis, Vol. 3, pp. 107-129.

Ming, Z., Nellippallil, A., Yan, Y., Wang, G., Goh, C., et al. (2017), "PDSIDES - A knowledge-based Platform for Decision Support in the Design of Engineering Systems", ASME 2017 International Design Engineering Technical Conferences and Computers and Information in Engineering Conference, Cleveland, Ohio.

Mistree, F., Muster, D., Shupe, J. and Allen, J. (1989), “A Decision-Based Perspective for the Design of Methods for Systems Design”, Recent Experiences in Multidisciplinary Analysis and Optimization, pp. 1111-1135.

Moeller, G. and Digman, L. (1981), “Operations Planning with VERT”, Operations Management, Vol. 29 No. 4, pp. 676-697.

Moullec, M.-L., Bouissou, M., Jankovic, M., Bocquet, J.-C., Réquillard, F. et al. (2013), "Toward System Architecture Generation and Performances Assessment Under Uncertainty Using Bayesian Networks", Journal of Mechanical Design, Vol. 135 No. 4, pp. 41002.

Murthy, V., Singh, V., Chen, T., Manmatha, R. and Comaniciu, D. (2016), "Deep Decision Network for Multiclass Image Classification”, IEEE Conference on Computer Vision and Pattern Recognition, pp. 2240-2248.

Panchal, J., Paredis, C., Allen, J. and Mistree, F. (2009), "Managing Design-Process Complexity: A Value-ofInformation Based Approach for Scale and Decision Decoupling", Journal of Computing and Information Science in Engineering, Vol. 9 No. 2, pp. 1-15.

Panchal, J., Sha, Z. and Kannan, K. (2017), "Understanding Design Decisions Under Competition Using Games With Information Acquisition and a Behavioral Experiment”, Journal of Mechanical Design, Vol. 139 No. 9 , pp. 91402.

Pandey, V. and Mourelatos, Z. (2015), “A New Method for Making Design Decisions: Decision Topologies", Journal of Mechanical Design, Vol. 137 No. 3, pp. 31401.

Pearl, J. (1988), Probabilistic Reasoning in Intelligent Systems: Networks of Plausible Inference, Morgan Kaufmann Publishers.

Renzi, C., Leali, F. and di Angelo, L. (2017), “A review on decision-making methods in engineering design for the automotive industry", Journal of Engineering Design, Vol. 28 No. 2, pp. 118-143.

Russel, S. and Norvig, P. (2010), Artificial Intelligence: A Modern Approach, 3rd ed., Prentice Hall.

Sen, C., Ameri, F. and Summers, J. (2010), "An Entropic Method for Sequencing Discrete Design Decisions", Journal of Mechanical Design, Vol. 132 No. 10, pp. 101004.

Sen, P. and Yang, J. (2012), Multiple Criteria Decision Support in Engineering Design, Springer, London.

Sha, Z. and Panchal, J.H. (2016), "A degree-based decision-centric model for complex networked systems", ASME 2016 International Design Engineering Technical Conferences and Computers and Information in Engineering Conference, Charlotte, NC, USA, August 21-24, 2016. https://doi.org/10.1115/DETC2016-60036

Shachter, R.D. (2016), "Decisions and Dependence in Influence Diagrams", Proceedings of the Eighth International Conference on Probabilistic Graphical Models, 2016, pp. 462-473.

Silver, M. and De Weck, O. (2007), "Time-Expanded Decision Networks: A Framework for Designing Evolvable Complex Systems", Systems Engineering, Vol. 10 No. 2, pp. 167-186.

Tappeta, R. and Renaud, J. (2001), "Interactive Multiobjective Optimization Design Strategy for Decision Based Design”, Journal of Mechanical Design, Vol. 123 No. 2, pp. 205-215.

Thompson, S. and Paredis, C. (2010), "An Investigation Into the Decision Analysis of Design Process Decisions", Journal of Mechanical Design, Vol. 132 No. 12, pp. 121009.

Ullman, D. (2006), Making Robust Decisions: Decision Management for Technical, Business, and Service Teams, Trafford Publishing.

Ullman, D.G., Herling, D. and D’Ambrosio, B. (1997), "What to do next: Using problem status to determine the course of action", Research in Engineering Design, Vol. 9 No. 4, pp. 214-227.

Dr. Mohammad Hassannezhad, Research Associate

University of Cambridge, Department of Engineering

Trumpington Street, CB2 1PZ Cambridge, United Kingdom

Email: mh844@eng.cam.ac.uk 